Bulletin of the Section of Logic

Volume 46:1/2 (2017), pp. 111-133

http://dx.doi.org/10.18778/0138-0680.46.1.2.09

Andrzej Pietruszczak

\title{
ON THESES WITHOUT ITERATED MODALITIES OF MODAL LOGICS BETWEEN C1 AND S5. PART 1
}

\begin{abstract}
This is the first, out of two papers, in which we identify all logics between C1 and S5 having the same theses without iterated modalities. All these logics can be divided into certain groups. Each such group depends only on which of the following formulas are theses of all logics from this group: (N), (T), (D), $\ulcorner$ (T) $\square q\urcorner$, and for any $n>0$ a formula $\left\ulcorner(\mathrm{T}) \vee\left(\mathrm{al} \mathrm{t}_{\mathrm{n}}\right)\right\urcorner$, where $(\mathrm{T})$ has not the atom ' $q$ ', and $(\mathrm{T})$ and $\left(\mathrm{alt}_{\mathrm{n}}\right)$ have no common atom. We generalize Pollack's result from [12], where he proved that all modal logics between S1 and S5 have the same theses which does not involve iterated modalities (i.e., the same first-degree theses).

Keywords: first-degree theses of modal logics; theses without iterated modalities; Pollack's theory of Basic Modal Logic; basic theories for modal logics between $\mathrm{C} 1$ and S5.
\end{abstract}

\section{Introduction}

A modal formula involves iterated modalities ${ }^{1}$ iff some instance of ' $\square$ ' or ' $\diamond$ ' (necessity and possibility signs, respectively) in it occurs within the scope of some other instance of ' $\square$ ' or ' $\nabla$ '. The iteration of modal operators leads to philosophical controversies. In [12, p. 355] we read:

As anyone who is familiar with the literature knows, there is a great deal of controversy concerning which, if any, of the extant theories of propositional modal logic correctly formalizes the logic of certain logical concepts such as analyticity and logical

\footnotetext{
${ }^{1}$ In Section 2 we recall some chosen facts concerning modal classical logics.
} 
necessity. Most of the controversy concerns certain principles that involve iterated modalities (where one modal operator occurs within the scope of another). For example, there is considerable disagreement about whether the principle $(\square p \supset \square \square p)$ [or: $\diamond \diamond p \supset \diamond p$ ] should be considered valid. However, when philosophers and logicians apply modal logic to concrete problems, they rarely need principles which involve iterated modalities. For most practical purposes, principles involving only one layer of modalities are all that are needed. This suggests that if we try to construct a theory of modal logic in which there are no iterated modalities, we can avoid most of the controversy and still have a theory that is strong enough for all of the normal uses to which modal logic is put.

For example, suppose that the railway line does not come to a certain village $V$ (we write: $\neg q$ ). Then, naturally, it is not possible to reach $V$ by rail (we write: $\neg \checkmark p) .{ }^{2}$ However let us assume that it is possible to build a railway line to $V$ (we write: $\diamond q$ ). Then it seems right to accept the following strict implication: 'If there exists a railway line to $V$ then it is possible to reach to $V$ by rail' (we write: $\square(q \supset \diamond p)$ ). Thus, if we accept the schemas $\ulcorner\square(\varphi \supset \psi) \supset(\diamond \varphi \supset \diamond \psi)\urcorner$ and $\ulcorner\diamond \diamond \varphi \supset \diamond \varphi\urcorner$ as logically valid, then using only these schemas, and modus ponens - by the premises ' $\diamond q$ ' and ' $\square(q \supset \diamond p)$ ' we obtain ' $\nabla p$ '. This will imply that now we can reach $V$ by rail, which contradicts the original claim (i.e., $\neg \diamond p$ ). So should we say second premiss is false? What kind of reasons can we find for that? Or should we rather question whether ' $\diamond \diamond p \supset \diamond p$ ' is logically valid? The following questions can make us think so: Should both instances of the operator ' $\nabla$ ' in the formula ' $\triangle \nabla p$ ' be read in the same way? If not, then should the formula ' $\diamond \diamond p$ ' be understood as: It is possible that it WILL BE possible that $p$ ? If so, then the way of reading a given instance of the operator ' $\diamond$ ' in a given formula depends on its position in the formula. Then (4) will not be logically valid. Thus - as Pollack wrote - if we reject formulas which involve iterated modalities, we can avoid such problems.

Even if we take no interest in the philosophical issues, then it is still intriguing to identify all groups of logics between $\mathbf{C 1}$ and S5 having the same theses without iterated modalities and to examine which theses of a given logic determine its belonging to a given group.

\footnotetext{
${ }^{2}$ In the example above modalities are understood as metaphysical (not logical).
} 
In [12] Pollack showed that all logics between S1 and S5 have exactly one "theory of modal logic in which there are no iterated modalities", i.e., all such modal logics have the same theses in which there are no iterated modalities. This theory Pollack called the theory B of "Basic Modal Logic". In Section 3 we will show that also all logics between Lemmon's logic S0.5 and S5 have the same "Basic Modal Logic".

Moreover, in this paper we will identify all groups of logics between C1 and S5 having the same theses without iterated modalities. We prove that each such group depends only on which of the following formulas are theses of all logics from this group:

$$
\begin{aligned}
& \square(p \supset p) \\
& \square p \supset p \\
& \square p \supset \diamond p \\
& (\square p \supset p) \vee \square q \\
& (\square p \supset p) \vee \square q_{1} \vee \square\left(q_{1} \supset q_{2}\right) \vee \cdots \vee \square\left(\left(q_{1} \wedge \cdots \wedge q_{n}\right) \supset q_{n+1}\right) \quad \text { for } n>0
\end{aligned}
$$

$\left(\right.$ Talt $\left._{n}\right)$

In the above formulas $\left(\right.$ Talt $\left._{0}\right)$ and $\left(\right.$ Talt $\left._{n}\right)$ we used as disjuncts the following formulas:

$$
\square q_{1} \vee \bigvee_{i=1}^{n} \square\left(\bigwedge_{j=1}^{i} q_{j} \supset q_{i+1}\right) \quad \text { for } n>0
$$

We will use $\left(a l t_{0}\right):=(\mathrm{Q})$. So $\left(\mathrm{Talt}_{0}\right)=\left\ulcorner(\mathrm{T}) \vee\left(\mathrm{al} \mathrm{t}_{0}\right)\right\urcorner$ and, for example, we have:

$$
\begin{gathered}
(\square p \supset p) \vee \square q_{1} \vee \square\left(q_{1} \supset q_{2}\right) \\
(\square p \supset p) \vee \square q_{1} \vee \square\left(q_{1} \supset q_{2}\right) \vee \square\left(\left(q_{1} \wedge q_{2}\right) \supset q_{3}\right)
\end{gathered}
$$

The formulas $(\mathrm{T})$ and $(\mathrm{Q})$ are adequate, respectively, for all reflexive Kripke frames and for all Kripke frames in which each point has no alternative. So $\left(\mathrm{Talt}_{0}\right)$ is adequate for all quasi-reflexive Kripke frames (i.e., for frames of the form $\langle W, R\rangle$ in which for each point $x \in W$ either $x R x$ or $\operatorname{Card}\{w \in W: x R w\}=0)$. Note that for any $n>0$, the formula $\left(\operatorname{alt}_{\mathrm{n}}\right)$ is adequate for Kripke frames in which each point has $\leqslant n$ alternatives (see, e.g., [15, p. 52]). So, for any $n \geqslant 0$, the formula $\left(\right.$ Talt $\left._{\mathrm{n}}\right)$ is adequate for Kripke frames $\langle W, R\rangle$ in which for each point $x \in X$ either $x R x$ or $\operatorname{Card}\{w \in W: x R w\} \leqslant n$. 
There are two reasons to limit our investigations only to the logics included in the logic S5. First, in S5 there is a «complete reduction» of iterated modalities, i.e., for any modal operator $O \in\{\square, \diamond\}$ and for any finite sequence $\mathcal{M}$ of modal operators, the formula $\ulcorner O \varphi \equiv \mathcal{M} O \varphi\urcorner$ is a thesis of S5. Of course, this reduction does not solve the problem of interpretation of iterations of modal operators.

Remark 1.1. The above note corresponds with the following observation. Sufficient for the semantic study of $\mathbf{S 5}$ are the models of the form $\langle W, V\rangle$, where $W$ is a non-empty set of worlds and $V$ is a function which to any pair built out of a formula and a world assigns a truth-value which preserves classical conditions for truth-value operators and for any $\varphi \in$ For and $x \in W$ we have:

$\left(V_{W}^{\square}\right) \quad V(\square \varphi, x)=1 \quad$ iff $\quad \forall_{y \in W} V(\varphi, y)=1$, $\left(V_{W}^{\diamond}\right) \quad V(\diamond \varphi, x)=1 \quad$ iff $\quad \exists_{y \in W} V(\varphi, y)=1$.

So, for example, for any $x \in W: V(\diamond \square p, x)=1$ iff $\exists_{y \in W} V(\square p, y)=1$ iff $\exists_{y \in W} \forall_{z \in W} V(p, z)=1$. It would not be possible to express this condition if one were to use a version of predicate logic where «empty binding» of quantifiers is forbidden. This version has two kinds of variables - free and bound (see, e.g., [16]). Only by changing a free variable to a bound one we can add the quantifier that binds the latter. Does this not call into question the coherence/possibility of iterations of modal operators in the $\operatorname{logic} \mathbf{S 5}$ ?

Second, all formulas that are not theses of S5 (for example, (Q)) are not associated with the problems raised by Pollack in [12].

\section{Preliminaries on modal logic}

\subsection{Basic notions and facts}

Classical and modal formulas. Let At be the set of all atoms (i.e., propositional letters): ' $p_{1}$ ', ' $q_{1}$ ', ' $p_{2}$ ', ' $q_{2}$ ', ' $p_{3}$ ', ' $q_{3}$ ', $\ldots$ (for ' $p_{1}$ ' and ' $q_{1}$ ', we use ' $p$ ' and ' $q$ ', respectively). The set $\mathbf{F o r}_{\mathrm{cl}}$ of all classical propositional formulas is formed in the standard way from atoms, brackets, and truth-value operators: ' $\neg$ ', ' $\vee$ ', ' $\wedge$ ', ' $\supset$ ', and ' $\equiv$ ' (connectives of negation, disjunction, conjunction, material implication, and material equivalence, respectively). The set For of all formulas for (propositional) modal logics is formed standardly from atoms, brackets, truth-value operators, and the 
modal operator ' $\square$ ' (the necessity sign; the possibility sign ' $\vee$ ' is the abbreviation of ' $\neg \square \neg$ '). A formula from For is called first-degree iff either it is classical or it is modal, but does not involve iterated ' $\square$ '. Let ${ }^{\mathbf{1}} \mathbf{F}$ or be the set of all first-degree formulas. That is, ${ }^{1}$ For is the smallest set $F$ such that: For $_{\mathrm{cl}} \subseteq \mathbf{F}$; for any $\varphi \in$ For $_{\text {cl }}$ we have $\ulcorner\square \varphi\urcorner \in F$; and for all $\varphi, \psi \in F$ we have $\ulcorner\neg \varphi\urcorner \in F$ and $\ulcorner(\varphi \circ \psi)\urcorner \in F$, for $\circ \in\{\wedge, \vee, \supset, \equiv\}$. Of course, For $_{\mathbf{c l}} \subsetneq{ }^{\mathbf{1}}$ For $\subsetneq$ For. For any subset $\Phi$ of For we put ${ }^{\mathbf{1}} \Phi:=\Phi \cap{ }^{\mathbf{1}}$ For.

We also put $\square \Phi:=\{\square \varphi: \varphi \in \Phi\}$, for any set $\Phi \subseteq$ For. Moreover, for any $k>0$ and any formula of the form $\left\ulcorner\alpha_{1} \wedge \cdots \wedge \alpha_{k}\right\urcorner$ (resp. $\left\ulcorner\alpha_{1} \vee \cdots \vee\right.$ $\left.\left.\alpha_{k}\right\urcorner\right)$ we write $\bigwedge\left\{\alpha_{1}, \ldots, \alpha_{k}\right\}$ or $\bigwedge_{i=1}^{k} \alpha_{i}$ (resp. $\bigvee\left\{\alpha_{1}, \ldots, \alpha_{k}\right\}$ or $\bigvee_{i=1}^{k} \alpha_{i}$ ). Finally, we put $\top:={ }^{\prime} p_{2} \supset p_{2}$ ' and $\perp:={ }^{\prime} p_{2} \wedge \neg p_{2}$ '.

A formula from ${ }^{\mathbf{1}}$ For is said to be in Modal Conjunctive Normal Form iff it is a conjunction (possibly degenerated), each conjunct of which is a disjunction (possibly degenerated) of formulas that belong either to $\mathbf{F o r}_{\mathbf{c l}}$ or to the set of all formulas of the form $\ulcorner\square \alpha\urcorner$ or $\ulcorner\diamond \beta\urcorner$, where $\alpha, \beta \in \mathbf{F o r}_{\mathbf{c l}}$ (see, e.g., [4, pp. 96-97]). Let MCNF be the set of all such formulas.

Taut and PL. PL-valuations (resp. cl-valuations) are assignments from For (resp. For cl $_{\text {) }}$ into $\{0,1\}$ fulfilling classical conditions for truth-value operators. Note that all formulas of the form $\ulcorner\square \varphi\urcorner$ have arbitrary values for all PL-valuations.

For any PL-valuation (resp. cl-valuation) $V$, if $V(\alpha)=1$ then we say that $\alpha$ is true in $V$. Let Taut be the set of classical tautologies, i.e., formulas from $\mathbf{F o r}_{\mathbf{c l}}$ which are true in all cl-valuations. Moreover, let $\mathbf{P L}$ be the set of all formulas which are true in all PL-valuations, i.e., the set of formulas which are instances of classical tautologies. Of course, we have: Taut $=$ For $_{\mathrm{cl}} \cap \mathrm{PL}$ and Taut $\subsetneq^{\mathbf{1}} \mathbf{P L} \subsetneq \mathbf{P L}$.

Modal logics. A subset $\boldsymbol{\Lambda}$ of For is a modal logic iff Taut $\subseteq \boldsymbol{\Lambda}$ and $\boldsymbol{\Lambda}$ is closed under two rules: detachment for material implication (modus ponens) and uniform substitution. Thus, by uniform substitution, all modal logics include the set PL. Moreover, this set is the smallest modal logic.

For any modal logic $\boldsymbol{\Lambda}$ and any set $\Phi$ of formulas we write $\boldsymbol{\Lambda}+\Phi$ to denote the smallest modal logic including the set $\boldsymbol{\Lambda} \cup \Phi$. Thus, the logic $\boldsymbol{\Lambda}+\Phi$ is the closure of $\boldsymbol{\Lambda} \cup \operatorname{sub}(\Phi)$ under modus ponens, where $\operatorname{sub}(\Phi)$ is the set of all substitution instances of formulas from $\Phi$, i.e., $\operatorname{sub}(\Phi):=\{\psi \in$ For : $\left.\exists_{\varphi \in \Phi} \psi=\operatorname{sub}(\varphi)\right\}$. Notice that $\boldsymbol{\Lambda}+\Phi$ may not be closed under other rules under which $\boldsymbol{\Lambda}$ is closed. To simplify the naming of logics, for a set $\Phi=\left\{\varphi_{1}, \ldots, \varphi_{k}\right\}$ of formulas we write $\boldsymbol{\Lambda}\left[\varphi_{1}, \ldots, \varphi_{k}\right]$ to denote $\boldsymbol{\Lambda}+\Phi$. 
For any logics $\boldsymbol{\Lambda}_{1}$ and $\boldsymbol{\Lambda}_{2}$, the set $\boldsymbol{\Lambda}_{1} \cap \boldsymbol{\Lambda}_{2}$ is also a logic. Note that we have the following (stronger) version of Theorem 2 from [5]:

Theorem 2.1. Let $\boldsymbol{\Lambda}$ be a logic and let $\varphi, \psi \in$ For be such that $\varphi$ and $\psi$ have no atoms in common and $\ulcorner\varphi \vee \psi\urcorner \in \boldsymbol{\Lambda}$. Then $\boldsymbol{\Lambda}=\boldsymbol{\Lambda}[\varphi] \cap \boldsymbol{\Lambda}[\psi]$.

\subsection{Normal and regular modal logics}

Formalization. A modal $\operatorname{logic} \boldsymbol{\Lambda}$ is normal iff $\boldsymbol{\Lambda}$ contains the following formula:

$$
\square(p \supset q) \supset(\square p \supset \square q)
$$

and is also closed under the necessity rule:

$$
\text { if } \varphi \in \boldsymbol{\Lambda} \text { then }\ulcorner\square \varphi\urcorner \in \boldsymbol{\Lambda} \text {. }
$$

So $\boldsymbol{\Lambda}$ is normal iff $\boldsymbol{\Lambda}$ contains (K) and (N), and is closed under the monotonicity rule:

$$
\text { if }\ulcorner\varphi \supset \psi\urcorner \in \boldsymbol{\Lambda} \text { then }\ulcorner\square \varphi \supset \square \psi\urcorner \in \boldsymbol{\Lambda} \text {. }
$$

Moreover, $\boldsymbol{\Lambda}$ is normal iff $\boldsymbol{\Lambda}$ contains (N) and is closed under the regularity rule:

$$
\text { if }\left\ulcorner\left(\varphi_{1} \wedge \varphi_{2}\right) \supset \psi\right\urcorner \in \boldsymbol{\Lambda} \text { then }\left\ulcorner\left(\square \varphi_{1} \wedge \square \varphi_{2}\right) \supset \square \psi\right\urcorner \in \boldsymbol{\Lambda} .
$$

We remind that $\mathbf{K}$ is the smallest normal modal logic. For any normal logic $\boldsymbol{\Lambda}$ and any set $\Phi$ of formulas we write $\boldsymbol{\Lambda} \oplus \Phi$ to denote the smallest normal logic including the set $\boldsymbol{\Lambda} \cup \Phi$. To simplify the naming of normal logics, for any formulas $\left(\mathrm{X}_{1}\right), \ldots,\left(\mathrm{X}_{\mathrm{k}}\right)$, the smallest normal logic including all of these formulas we denote by $\mathbf{K X}_{1} \ldots \mathbf{X}_{\mathrm{k}}$.

For defining other logics we will also make use of the following formulas:

$$
\begin{gathered}
p \supset \square \diamond p \\
\square p \supset \square \square p \\
\vee p \supset \square \diamond p
\end{gathered}
$$

We put $\mathbf{T}:=\mathbf{K T}, \mathbf{S} 4:=\mathbf{K T} \mathbf{4}$, and $\mathbf{S 5}:=\mathbf{K T} \mathbf{5}$. We have $\mathbf{K T}=\mathbf{K D} \oplus$ $\left(\mathrm{Talt}_{0}\right)$ and $\mathbf{K B} 4=\mathbf{K B 5}=\mathbf{K B} 45=\mathbf{K 5} \oplus\left(\mathrm{Talt}_{0}\right)=\mathbf{K} 4 \mathbf{5} \oplus\left(\mathrm{Talt}_{0}\right)$. So S5 $:=$ KT5 $=$ KD5 $\oplus\left(\right.$ Talt $\left._{0}\right)=$ KTB4 $=$ KDB5 $=$ KDB4 $=$ KD45 $\oplus\left(\right.$ Talt $\left._{0}\right)$. These facts we can get syntactically or semantically (see, e.g., p. 120). 
The following lemma will be used in the proofs of Fact 2.19 and facts 5.1 and 5.2 in Part 2. It is taken from [12, Theorem 2] and it can be proved following [4, pp. 105-107].

Lemma 2.2. For any $k \geqslant 0$ and $\alpha, \beta, \gamma_{1}, \ldots, \gamma_{k} \in$ For $_{\mathrm{cl}}$ :

1. $\left\ulcorner\alpha \vee \diamond \beta \vee \bigvee_{i=1}^{k} \square \gamma_{j}\right\urcorner \in \mathbf{S 5}$ iff either $\ulcorner\alpha \vee \beta\urcorner \in$ Taut or $\left\ulcorner\beta \vee \gamma_{j}\right\urcorner \in$ Taut, for some $j \in\{1, \ldots, k\}$.

2. $\left\ulcorner\alpha \vee \bigvee_{i=1}^{k} \square \gamma_{j}\right\urcorner \in \mathbf{S 5}$ iff either $\alpha \in$ Taut or $\gamma_{j} \in$ Taut, for some $j \in\{1, \ldots, k\}$.

3. $\left\ulcorner\diamond \beta \vee \bigvee_{j=1}^{k} \square \gamma_{j}\right\urcorner \in \mathbf{S 5}$ iff either $\beta \in$ Taut or $\left\ulcorner\beta \vee \gamma_{j}\right\urcorner \in$ Taut, for some $j \in\{1, \ldots, k\}$.

4. $\left\ulcorner\bigvee_{j=1}^{k} \square \gamma_{j}\right\urcorner \in \mathbf{S 5}$ iff $\gamma_{j} \in$ Taut, for some $j \in\{1, \ldots, k\}$.

A modal logic is regular iff it closed under the rule (RR). So a logic is regular iff it contains $(\mathrm{K})$ and is closed under (RM). Moreover, a logic is normal iff it is regular and contains $(\mathrm{N})$. By $(\mathrm{K})$ and $(\mathrm{RR})$, for any regular $\operatorname{logic} \boldsymbol{\Lambda}$ and any $k \geqslant 1$ we obtain that:

$$
\text { if }\left\ulcorner\left(\varphi_{1} \wedge \cdots \wedge \varphi_{k}\right) \supset \psi\right\urcorner \in \boldsymbol{\Lambda} \text { then }\left\ulcorner\left(\square \varphi_{1} \wedge \cdots \wedge \square \varphi_{k}\right) \supset \square \psi\right\urcorner \in \boldsymbol{\Lambda}
$$

We remind that $\mathbf{C 2}$ is the smallest regular modal logic. Moreover, E2 and D2 are the smallest regular logics which contain (T) and (D), respectively (see, e.g., $[6,7,14,15])$. Lemmon's logic E3 is the smallest logic which is closed under (RM) and contains $(\mathrm{T})$ and the following stronger version of $(\mathrm{K})$ :

$$
\square(p \supset q) \supset \square(\square p \supset \square q)
$$

Of course, the logic E3 is regular, since it contains (K).

We say that a regular logic is properly regular iff it is not normal. Of course, a regular logic $\boldsymbol{\Lambda}$ is properly regular iff $\boldsymbol{\Lambda} \cap \square$ For $=\varnothing$ iff (N) $\notin \boldsymbol{\Lambda}$. The logics C2, D2, E2, and E3 are properly regular.

As for normal logics, also for any regular logic $\boldsymbol{\Lambda}$ and any set $\Phi$ of formulas we write $\boldsymbol{\Lambda} \oplus \Phi$ to denote the smallest regular logic including the set $\boldsymbol{\Lambda} \cup \Phi$. (Of course, if $\boldsymbol{\Lambda}$ is normal, i.e., if (N) $\in \boldsymbol{\Lambda}$, then $\boldsymbol{\Lambda} \oplus \Phi$ is normal.) Also to simplify naming of regular logics, for any formulas $\left(\mathrm{X}_{1}\right)$, $\ldots,\left(\mathrm{X}_{\mathrm{k}}\right)$, the smallest regular logic including all of these formulas we denote by $\mathbf{C x}_{1} \ldots \mathrm{X}_{\mathrm{k}}$. We put $\mathbf{D 2}:=\mathbf{C D}, \mathbf{E 2}:=\mathbf{C T}=\mathbf{D} 2 \oplus\left(\right.$ Talt $\left._{0}\right)$, and $\mathbf{E 3}:=\mathbf{C T} \oplus(\mathrm{sK})$.

Note that all regular logics having a thesis (N) are closed under the rule (RN). Hence all regular logics which have theses (5) and either $\ulcorner\diamond \top\urcorner$, 
or (D), or (T) are closed under (RN). So, e.g., we have $\mathbf{C T 5}=\mathbf{K T} \mathbf{5}=\mathbf{S 5}$, CD5 = KD5, and CD45 = KD45. Thus, to avoid «normalization» of regular logics one has to use some special formulas. We adopt a convention from $\left[15\right.$, p. 206] and for any formula $\chi$ we put $\chi^{(1)}:=\ulcorner N \supset \chi\urcorner$. Note that in all regular logics, any formula of the form $\ulcorner\square \varphi \supset \psi\urcorner$ is equivalent to $\ulcorner\mathrm{N} \supset(\square \varphi \supset \psi)\urcorner$. Thus, the formulas (T), (D), (sK), and (4) are equivalent to $\left(\mathrm{T}^{(1)}\right),\left(\mathrm{D}^{(1)}\right),\left(\mathrm{sK}^{(1)}\right)$, and $\left(4^{(1)}\right)$, respectively.

We put $\mathrm{N}^{1}:=\ulcorner\mathrm{N} \supset \square \mathrm{N}\urcorner$ and $\mathrm{F}:=\ulcorner\diamond \perp\urcorner$. The properly regular logic CF is called Falsum; for any $\varphi \in$ For we have $\ulcorner\Delta \varphi\urcorner \in \mathbf{C F}$. We have (see [15, vol. II, Corollary 2.4]):

$$
\mathrm{CN}^{1} \mathrm{X}_{1}^{(1)} \ldots \mathrm{X}_{\mathrm{k}}^{(1)}=\mathbf{C F} \cap \mathrm{KX}_{1} \ldots \mathrm{X}_{\mathrm{k}} .
$$

Thus, e.g., $\mathbf{C F} \cap \mathrm{KD} 45=\mathrm{CN}^{1} \mathbf{D}^{(1)} \mathbf{4}^{(1)} \mathbf{5}^{(1)}=\mathrm{CN}^{\mathbf{1}} \mathbf{D} 45^{(1)}=\mathbf{C D} 45^{(1)}$, since $\mathrm{N}^{1}$ is an instance of (4). Similarly, $\mathbf{C F} \cap \mathbf{S} 4=\mathbf{C F} \cap \mathbf{K T} \mathbf{4}=\mathbf{C N}^{\mathbf{1}} \mathbf{T} \mathbf{4}=$ $\mathbf{C T} 4$ and $\mathbf{C F} \cap \mathbf{S 5}=\mathbf{C F} \cap \mathbf{K T 5}=\mathbf{C T 5}{ }^{(1)}=\mathbf{E} 5$ in Kripke sense [5, p. 209 210]. Indeed, because $\ulcorner\square p \supset \mathrm{N}\urcorner \in \mathbf{C 2}$, we have $(4) \in \mathbf{C T 5}^{(1)}$; and so $\mathrm{CF} \cap \mathrm{S} 5=\mathrm{CF} \cap \mathrm{KT} 5=\mathrm{CF} \cap \mathrm{KT} 45=\mathrm{CN}^{1} \mathbf{T} 45^{(1)}=\mathrm{CT}_{4} 5^{(1)}=\mathrm{CT}^{(1)}$.

Kripke semantics. For the semantical analysis of normal logics we may use standard normal Kripke frames of the form $\langle W, R\rangle$, where $W$ is a nonempty set of worlds and $R$ is a binary accessibility relation in $W$. For any Kripke frame $\langle W, R\rangle$ a model is any triple $\langle W, R, V\rangle$, where $V$ is a function which to any pair built out of a formula and a world assigns a truth-value with respect to $R$. More precisely, $V:$ For $\times \mathbf{W} \rightarrow\{\mathbf{0}, \mathbf{1}\}$ preserves classical conditions for truth-value operators and for any $\varphi \in$ For and $x \in W$ we have:

$\left(\mathrm{n} V_{R}^{\square}\right) \quad V(\square \varphi, x)=1 \quad$ iff $\quad \forall_{y \in R[x]} V(\varphi, y)=1$, where for any $x \in W$ we put $R[x]:=\{y \in W: x R y\}$.

For the semantical analysis of properly regular logics we may use regular Kripke frames of the form $\langle W, R, N\rangle$, where $W$ and $R$ are the same as for normal frames and $N$ is a set of normal worlds. For any Kripke frame $\langle W, R, N\rangle$ a model is any quadruple $\langle W, R, N, V\rangle$, where $V$ is a function from For $\times \mathbf{W}$ into $\{0,1\}$ which preserves classical conditions for truthvalue operators and for any $\varphi \in$ For and $x \in W$ we have:

$\left.\left(\mathrm{r} V_{R}^{\square}\right) \quad\right] V(\square \varphi, x)=1 \quad$ iff $\quad x \in N$ and $\forall_{y \in R[x]} V(\varphi, y)=1$.

Of course, regular frames and regular models of the form $\langle W, R, W\rangle$ and $\langle W, R, W, V\rangle$, respectively, i.e. with $N=W$, are suitable for normal logics and can be identified with $\langle W, R\rangle$ and $\langle W, R, V\rangle$, respectively. 
We say that a model $\langle W, R, V\rangle$ (resp. $\langle W, R, N, V\rangle)$ is based on the frame $\langle W, R\rangle$ (resp. $\langle W, R, N\rangle$ ). As usual, we say that a formula is $\varphi$ is true in a world $x$ of a model $\langle W, R, V\rangle$ (resp. $\langle W, R, N, V\rangle)$ iff $V(\varphi, x)=1$. We say that a formula is true in a model iff it is true in all worlds of this model. Next we say that a formula is true in a frame iff it is true in every model which is based on this frame. Moreover, for any modal logic $\boldsymbol{\Lambda}$ and any class $\mathscr{C}$ of frames (resp. models) we say that:

- a given formula is valid in $\mathscr{C}$ iff it is true in all members of $\mathscr{C}$;

- $\boldsymbol{\Lambda}$ is sound with respect to $\mathscr{C}$ iff all members of $\boldsymbol{\Lambda}$ are valid in $\mathscr{C}$;

- $\boldsymbol{\Lambda}$ is complete with respect to $\mathscr{C}$ iff all valid formulas in $\mathscr{C}$ are members of $\boldsymbol{\Lambda}$;

- $\boldsymbol{\Lambda}$ is determined by $\mathscr{C}$ iff $\boldsymbol{\Lambda}$ is sound and complete with respect to $\mathscr{C}$.

Let us recall that a binary relation $R$ in $W \times W$ (resp. a frame $\langle W, R\rangle$, a model $\langle W, R, V\rangle$ ) is called, respectively: (i) empty iff $R=\varnothing$; (ii) universal iff $R=W \times W$; (iii) reflexive iff $\forall_{x \in W} x R x$; (iv) serial iff $\forall_{x \in W} \exists_{y \in W} x R y$; (v) symmetric iff $\forall_{x, y \in W}(x R y \Rightarrow y R x)$; (vi) transitive iff $\forall_{x, y, z \in W}(x R y$ \& $y R z \Rightarrow x R z)$; (vii) Euclidean iff $\forall_{x, y, z \in W}(x R y \& x R z \Rightarrow y R z)$; (viii) quasireflexive iff $\forall_{x, y \in W}(x R y \Rightarrow x R x)$ iff $\forall_{x \in W}(x R x$ or $R[x]=\varnothing)$. Additionally we will consider two classes of relation (frames, models): for any $n \geqslant 0$, $(\mathrm{ix})_{n} \forall_{x \in W} \operatorname{Card} R[x] \leqslant n ;(\mathrm{x})_{n} \forall_{x \in W}(x R x$ or $\operatorname{Card} R[x] \leqslant n)$. Of course, the condition (ix) $)_{0}$ says that $R=\varnothing$ and $(\mathrm{x})_{0}$ says that $R$ is quasi-reflexive. Moreover, note that for any binary relation $R$ we have:

( $*$ ) $R$ is reflexive iff $R$ is serial and quasi-reflexive.

( $\star$ ) $R$ is symmetric and transitive iff $R$ is symmetric and Euclidean iff $R$ is Euclidean and quasi-reflexive.

For the individual formulas we can assign the respective kinds of frames. We have the following pairs: (Q) to emptiness; (T) to reflexivity; (B) to symmetry; (4) to transitivity; (5) to Euclideanness; (D) to seriality; (Talt 0 ) to quasi-reflexivity; $\left(\right.$ alt $\left._{\mathrm{n}}\right)$ to $(\mathrm{ix})_{n}$; and $\left(\right.$ Talt $\left._{\mathrm{n}}\right)$ to $(\mathrm{x})_{n}$, for any $n>0$.

The following theorem is standard (cf., e.g., [1, 15, 17]).

TheOREm 2.3. 1. The logics $\mathbf{K}$ and $\mathbf{S} 5$ are determined, respectively, by the class of all normal Kripke frames and by the class of all universal normal Kripke frames.

2. Let $\left(\mathrm{X}_{1}\right), \ldots,\left(\mathrm{X}_{\mathrm{k}}\right)$ be any formulas from among the following ones: (T), (D), (B), (4), (5), (Q), (Talt $\left.\mathrm{t}_{0}\right)$, and for any $n>0,\left(\mathrm{al} \mathrm{t}_{\mathrm{n}}\right)$ and $\left(\mathrm{Talt}_{\mathrm{n}}\right)$. Then the normal logic $\mathrm{KX}_{1} \ldots \mathrm{X}_{\mathrm{k}}$ is determined by the class 
of all normal Kripke frames which satisfy all conditions for formulas $\left(\mathrm{X}_{1}\right), \ldots,\left(\mathrm{X}_{\mathrm{k}}\right)$.

From the Theorem 2.3 and $(\star \star)$ we have: $\mathbf{K T}=\mathbf{K D} \oplus\left(\right.$ Talt $\left._{0}\right)$, and $\mathbf{K B 4}=\mathbf{K B 5}=\mathbf{K B} 45=\mathbf{K 5} \oplus\left(\mathrm{Talt}_{0}\right)=\mathbf{K 4 5} \oplus\left(\right.$ Talt $\left._{0}\right)$. So S5 $:=$ $\mathrm{KT5}=\mathrm{KD5} \oplus\left(\mathrm{Talt}_{0}\right)=\mathrm{KTB} 4=\mathrm{KDB} 5=\mathrm{KDB} 4=\mathrm{KD} 45 \oplus\left(\mathrm{Talt}_{0}\right)$.

The following theorem also is standard (see, e.g., [5, pp. 214-217] and [15, pp. 204-206]).

Theorem 2.4. 1. C2 is determined by the class of all regular Kripke frames.

2. The logic E2 (resp. D2; E3) is determined by the class of all reflexive (resp. serial; both reflexive and transitive) regular Kripke frames.

3. The logic $\mathbf{C F}$ is determined by the single regular Kripke frame $\langle\{w\}$, $\varnothing, \varnothing\rangle$, where $R=\varnothing=N$.

4. If a normal logic $\mathbf{K X}_{1} \ldots \mathrm{X}_{\mathrm{k}}$ is determined by a class $\mathscr{F}$ of normal Kripke frames then the properly regular logic $\mathbf{C N}^{1} \mathrm{X}_{1}^{(1)} \ldots \mathrm{x}_{\mathrm{k}}^{(1)}(=\mathbf{C F} \cap$ $\left.\mathrm{KX}_{1} \ldots \mathrm{X}_{\mathrm{k}}\right)$ is determined by the class of frames obtained by adding to $\mathscr{F}$ the single regular frame $\langle\{w\}, \varnothing, \varnothing\rangle$.

\subsection{The logics S1, S2, and S3}

For a formulation of the logic $\mathbf{S} 1$ we use the following abbreviations for so-called strict implication ' $\prec$ ' and strict equivalence ' $\succ \prec$ ', respectively. Namely, for all formulas $\varphi$ and $\psi$ we put: $\ulcorner\varphi \prec \psi\urcorner:=\ulcorner\square(\varphi \supset \psi)\urcorner$ and $\ulcorner\varphi \succ \prec \psi\urcorner:=\ulcorner(\varphi \prec \psi) \wedge(\psi \prec \varphi)\urcorner$. Moreover, we use also the following formula:

$$
((p \prec q) \wedge(q \prec r)) \supset(p \prec r)
$$

Following Lemmon [6, pp. 177-178] we say that a modal logic $\boldsymbol{\Lambda}$ is closed under substitutability of strict equivalents iff for all $\varphi, \psi, \chi \in$ For we have:

$$
\text { if }\ulcorner\varphi \succ \psi\urcorner \in \boldsymbol{\Lambda} \text { and } \chi \in \boldsymbol{\Lambda} \text {, then } \chi[\varphi / \psi] \in \boldsymbol{\Lambda}
$$

where $\chi[\varphi / \psi]$ is any formula that results from $\chi$ by replacing one or more occurrences of $\varphi$, in $\chi$, by $\psi$. Moreover, we say that a modal logic $\boldsymbol{\Lambda}$ is closed under Becker's rule iff for all $\varphi, \psi \in$ For we have:

$$
\text { if }\ulcorner\varphi \prec \psi\urcorner \in \boldsymbol{\Lambda} \text { then }\ulcorner\square \varphi \prec \square \psi\urcorner \in \boldsymbol{\Lambda} \text {. }
$$

Following Lemmon [6, pp. 177-178] the logics S1, S2, and S3 are the smallest modal logics that includes the set $\square$ Taut and, respectively: 
1. $\mathbf{S} 1$ is closed under (SSE), and contains the formulas (T), $\square(\mathrm{T})$, and $\square(\mathrm{X})$,

2. $\mathbf{S} 2$ is closed under (RB), contains ( $\mathrm{T}), \square(\mathrm{T})$, and $\square(\mathrm{K})$,

3. S3 contains the formulas $(\mathrm{T}), \square(\mathrm{T})$, and $\square(\mathrm{sK})$.

The logic S1 contains (X), (K), and $\square(\mathrm{K}){ }^{3} \quad$ The logic S2 contains (K), $(\mathrm{X})$, and $\square(\mathrm{X})$. The logic S3 is closed under (RB) and contains (K), (sK), and $\square(\mathrm{K})$. It is known that $\mathbf{S} \mathbf{5} \mathbf{S 2} \subsetneq \mathbf{S 3}$. For example, the formulas ' $\square(p \wedge q) \prec(\square p \wedge \square q)^{\prime}$, ' $(\square p \wedge \square q) \prec \square(p \wedge q)$ ' and ' $\nabla(p \wedge q) \prec \diamond p$ ' belong to $\mathbf{S 2}$, but they are not members of $\mathbf{S 1}$. Moreover, $(\mathrm{sK}), \square(\mathrm{sK}) \notin \mathbf{S 2}$.

As Kripke [5] and Lemmon [8] noted, $\mathbf{S 2}=\mathbf{E 2}[\mathrm{N}]$ and $\mathbf{S 3}=\mathbf{E} 3[\mathrm{~N}]$. Hence, in the light of Theorem 2.1, E2 $=\mathbf{S 2} \cap \mathbf{C F}$ and $\mathbf{E 3}=\mathbf{S 3} \cap \mathbf{C F}$, because ' $\neg \square q \vee \mathrm{N}$ ' belongs to $\mathbf{C 2}$ and $\mathbf{C F}=\mathbf{C 2}[\diamond \perp]=\mathbf{E 2}[\diamond \perp]=\mathbf{E} \mathbf{3}[\diamond \perp]$ (see [5, p. 210]). Moreover, for every $\varphi \in$ For: $\varphi \in \mathbf{E 2}$ iff $\ulcorner\square \varphi\urcorner \in \mathbf{S 2}$; $\varphi \in \mathbf{E} 3$ iff $\ulcorner\square \varphi\urcorner \in \mathbf{S 3}$ (cf. [5, p. 217]).

In [6] the logic $\mathbf{S} \mathbf{4}$ is described as the smallest modal logic which contains $(\mathrm{T})$ and $(\mathrm{sK})$, and is closed under $(\mathrm{RN})$. Of course, $\mathbf{S} 4$ contains $(\mathrm{K})$, $\square(\mathrm{K})$, and $\square(\mathrm{sK})$. We have that $\mathbf{S 3} \subsetneq \mathbf{S} 4$. For example $(4) \notin \mathbf{S 3}$.

For logics S2 and S3 we have similar determination theorems as for E2 and E3, respectively, but in this case truth in regular Kripke models is decided only in normal worlds (see, e.g., [5, pp. 210-211 and 214-217], and $[13$, p. 65$])$. Semantic investigations of $\mathbf{S} \mathbf{1}$ was presented by Chellas and Segerberg [2].

\subsection{Very weak t-normal and t-regular logics}

We say that a modal logic is t-regular ${ }^{4}$ iff it includes the following set:

$$
\mathbf{R}_{\text {Taut }}:=\left\{\left(\square \varphi_{\mathbf{1}} \wedge \square \varphi_{\mathbf{2}}\right) \supset \square \psi:\left\ulcorner\left(\varphi_{\mathbf{1}} \wedge \varphi_{\mathbf{2}}\right) \supset \psi\right\urcorner \in \text { Taut }\right\} .
$$

So a logic is t-regular iff it contains $(\mathrm{K})$ and includes the following set:

$$
\mathbf{M}_{\text {Taut }}:=\{\square \varphi \supset \square \psi:\ulcorner\varphi \supset \psi\urcorner \in \text { Taut }\} .
$$

By substitution all t-regular logics include the sets $\mathbf{R}_{\mathbf{P L}}:=\left\{\left(\square \varphi_{\mathbf{1}} \wedge \square \varphi_{\mathbf{2}}\right) \supset\right.$ $\left.\square \psi:\left\ulcorner\left(\varphi_{\mathbf{1}} \wedge \varphi_{\mathbf{2}}\right) \supset \psi\right\urcorner \in \mathbf{P L}\right\}$ and $\mathbf{M}_{\mathbf{P L}}:=\{\square \varphi \supset \square \psi:\ulcorner\varphi \supset \psi\urcorner \in \mathbf{P L}\}$.

\footnotetext{
${ }^{3}$ For the logic $\mathbf{S} 1$ see, e.g., [2, pp. 12-15], [3], and [9, pp. 290-292].

${ }^{4}$ For details concerning t-regular and t-normal logics (see, e.g., $[9,10,11]$ ).
} 
Moreover, by $\mathbf{R}_{\mathrm{PL}}$ and $(\mathrm{K})$, respectively, all t-regular logics contain (X) and for any $k \geqslant 1$ we obtain:

$$
\text { if }\left\ulcorner\left(\varphi_{1} \wedge \cdots \wedge \varphi_{k}\right) \supset \psi\right\urcorner \in \mathbf{P L} \text {, then }\left\ulcorner\left(\square \varphi_{1} \wedge \cdots \wedge \square \varphi_{k}\right) \supset \square \psi\right\urcorner \in \boldsymbol{\Lambda} \text {. }
$$

We say that a modal logic is t-normal iff it is t-regular and contains (N). So a logic is t-normal iff it contains (K) and includes the set $\square$ Taut. By substitution all t-normal logics include the set $\square \mathbf{P L}$.

A t-regular logic is properly t-regular iff it is not t-normal. So a tregular logic $\boldsymbol{\Lambda}$ is properly t-regular iff (N) $\notin \boldsymbol{\Lambda}$ iff $\boldsymbol{\Lambda} \cap \square$ For $=\varnothing$. We say that a t-regular (resp. t-normal) logic $\boldsymbol{\Lambda}$ is very weak iff every thesis of $\boldsymbol{\Lambda}$ is a substitution of some thesis of $\boldsymbol{\Lambda}$ without iterated modalities. For these logics all «relevant theses» belong to the set ${ }^{\mathbf{1}}$ For.

The smallest t-regular logic is denoted by C1. Moreover, E1 and D1 are the smallest t-regular logics which contain (T) and (D), respectively, i.e., $\mathbf{E} \mathbf{1}=\mathbf{C} \mathbf{1}[\mathrm{T}]$ and $\mathbf{D} \mathbf{1}=\mathbf{C} \mathbf{1}[\mathrm{D}]$ (see, e.g., $[6,14]$ ). These logics are properly t-regular.

The smallest t-normal logic is denoted by $\mathbf{S O . 5}^{\circ}$ (see [14]). Of course, $\mathbf{S 0 . 5 ^ { \circ }}=\mathbf{C 1}[\mathrm{N}]$ and $\mathbf{S 0 . 5 ^ { \circ }}[\mathrm{D}]=\mathbf{C 1}[\mathrm{N}, \mathrm{D}]$. The Lemmon's [6] logic S0.5 is the smallest t-normal logic containing $(\mathrm{T})$. Clearly, S0.5 $=\mathbf{S 0 . 5 ^ { \circ }}[\mathrm{T}]=$ C1[N, T]. In [11] it has been proved that for any $\varphi \in$ For: $\ulcorner\square \varphi\urcorner \in \mathbf{S 0 . 5 ^ { \circ }}$ iff $\varphi \in \mathbf{P L}$ iff $\ulcorner\square \varphi\urcorner \in \mathbf{S 0 . 5}$. We have S0.5 $\subsetneq \mathbf{S} 1$. For example, $\square(\mathrm{T}), \square(\mathrm{X}) \notin$ S0.5.

Every regular (resp. normal) logic is t-regular (resp. t-normal) and every modal logic which includes some t-regular (resp. t-normal) logic is also t-regular (resp. t-normal). Hence for any formulas $\left(\mathrm{X}_{1}\right), \ldots,\left(\mathrm{X}_{\mathrm{k}}\right)$, the logic $\mathbf{C 1}\left[\mathrm{X}_{1} \ldots \mathrm{X}_{\mathrm{k}}\right]$ (resp. S0.5 ${ }^{\circ}\left[\mathrm{X}_{1} \ldots \mathrm{X}_{\mathrm{k}}\right]$ ) is t-regular (resp. t-normal). Moreover, for any $\operatorname{logic} \boldsymbol{\Lambda}: \boldsymbol{\Lambda}$ is t-regular (resp. t-normal) and is included in S5 iff $\mathbf{C} \mathbf{1} \subseteq \boldsymbol{\Lambda} \subseteq \mathbf{S} 5$ (resp. S0.5 $\subseteq \boldsymbol{\Lambda} \subseteq \mathbf{S 5}$ ). Also note that for any t-regular (resp. t-normal) logics $\boldsymbol{\Lambda}_{1}$ and $\boldsymbol{\Lambda}_{2}$, the logic $\boldsymbol{\Lambda}_{1} \cap \boldsymbol{\Lambda}_{2}$ is t-regular (resp. t-normal).

From our definitions we obtain:

FACT 2.5. For any modal logic $\boldsymbol{\Lambda}$ and any set $\Phi \subseteq{ }^{\mathbf{1}}$ For:

1. $\mathbf{C} 1+\Phi \subseteq \boldsymbol{\Lambda}$ iff ${ }^{\mathbf{1}}(\mathbf{C} \mathbf{1}+\Phi) \subseteq{ }^{\mathbf{1}} \boldsymbol{\Lambda}$. So if ${ }^{\mathbf{1}}(\mathbf{C} \mathbf{1}+\Phi) \subsetneq{ }^{\mathbf{1}} \boldsymbol{\Lambda}$ then $\mathbf{C} \mathbf{1}+\Phi \subsetneq$ $\Lambda$.

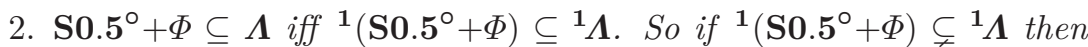
$\mathrm{S} 0.5^{\circ}+\Phi \subsetneq \Lambda$. 
Proof: Ad 1. " $\Rightarrow$ " Obvious. " $\Leftarrow$ " If ${ }^{\mathbf{1}}(\mathbf{C} 1+\Phi) \subseteq{ }^{\mathbf{1}} \boldsymbol{\Lambda}$ then $\mathbf{R}_{\text {Taut }} \cup \Phi \subseteq$ ${ }^{1} \boldsymbol{\Lambda} \subseteq \boldsymbol{\Lambda}$. Hence $\boldsymbol{\Lambda}$ is t-regular and includes $\Phi$. So $\mathbf{C} \mathbf{1}+\Phi \subseteq \boldsymbol{\Lambda}$.

Ad 2. " $\Rightarrow$ " Obvious. " $\Leftarrow$ " If ${ }^{\mathbf{1}}\left(\mathbf{S 0} .5^{\circ}+\Phi\right) \subseteq{ }^{\mathbf{1}} \boldsymbol{\Lambda}$ then $\square$ Taut $\cup\{(\mathrm{K}),(\mathrm{N})\} \cup$ $\Phi \subseteq{ }^{\mathbf{1}} \boldsymbol{\Lambda} \subseteq \boldsymbol{\Lambda}$. Hence $\boldsymbol{\Lambda}$ is t-normal and includes $\Phi$. So S0.5 $+\Phi \subseteq \boldsymbol{\Lambda}$.

Notice that $\mathbf{C} \mathbf{1}$ contains the following formulas:

$$
\begin{aligned}
\square p & \equiv \neg \vee \neg p \\
\square(p \supset q) & \supset(\diamond p \supset \diamond q) \\
\square(p \wedge q) & \equiv(\square p \wedge \square q) \\
\diamond(p \vee q) & \equiv(\diamond p \vee \diamond q) \\
\diamond(p \supset q) & \equiv(\square p \supset \diamond q) \\
(\square p \vee \square q) & \supset \square(p \vee q)
\end{aligned}
$$

Since $\left(R^{\diamond}\right)$ belongs to $\mathbf{C} 1$, so in all t-regular logics the formula (D) may be replaced by $\ulcorner\diamond \top\urcorner$. Hence $\mathbf{D} \mathbf{1}=\mathbf{C 1}[\mathrm{D}]=\mathbf{C 1}[\diamond \top]$ and $\mathbf{S 0 . 5 ^ { \circ }}[\mathrm{D}]=$ S0.5 ${ }^{\circ}[\diamond \top]=\mathbf{C 1}[\mathrm{N}, \diamond \top]$.

By using uniform substitution, (R), and the set $\mathbf{R}_{\mathbf{P L}}$, we can prove: Lemma $2.6([9,10,11])$. In any $t$-regular logic (Talt $\left.t_{0}\right)$ may be replaced by any of the following formulas: ${ }^{5}$

$$
\begin{aligned}
& \square p \supset(p \vee \square q) \quad \nabla q \supset(\square p \supset p) \\
& (\square p \supset p) \vee \square \perp \quad \diamond \top \supset(\square p \supset p) \\
& \square p \supset(p \vee \square \perp) \quad \square p \supset(p \vee \square \neg p) \quad \square p \supset(p \vee \square \neg p \vee \square \perp)
\end{aligned}
$$

So $\mathbf{E} 1=\mathbf{C 1}\left[\diamond \top, \mathrm{T}_{\mathrm{q}}\right]=\mathbf{C 1}\left[\mathrm{D}, \mathrm{T}_{\mathrm{q}}\right]=\mathbf{C 1}\left[\diamond \top, \mathrm{Talt}_{0}\right]=\mathbf{C 1}\left[\mathrm{D}, \mathrm{Talt}_{0}\right]$ and S0.5 $=\mathbf{S 0 . 5 ^ { \circ }}\left[\mathrm{D}, \mathrm{T}_{\mathrm{q}}\right]=\mathbf{S 0 . 5 ^ { \circ }}\left[\mathrm{D}, \mathrm{Talt}_{0}\right]$. Moreover, by definitions and Theorem 2.1, we obtain:

FACT 2.7. For any $n \geqslant 0$ :

1. $\mathbf{C} 1\left[\mathrm{Talt}_{\mathrm{n}}\right]=\mathbf{E} \mathbf{1} \cap \mathbf{C} \mathbf{1}\left[\mathrm{alt} \mathrm{t}_{\mathrm{n}}\right]=\mathbf{S} \mathbf{0 . 5} \cap \mathbf{C} \mathbf{1}\left[\mathrm{al} \mathrm{t}_{\mathrm{n}}\right]$ and

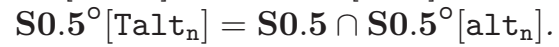

2. $\mathbf{C} 1=\mathbf{D} 1 \cap \mathbf{C} 1\left[\mathrm{alt}_{\mathrm{n}}\right]=\mathbf{D} \mathbf{1} \cap \mathbf{C} \mathbf{1}\left[\mathrm{Talt}_{\mathrm{n}}\right]$ and

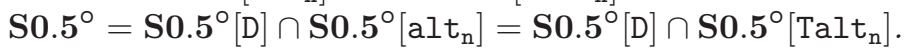

Proof: $A d$ 1. Since $\mathbf{E} \mathbf{1}=\mathbf{C 1}[\mathrm{T}]$ and $\mathbf{S 0 . 5}=\mathbf{S 0 . 5 ^ { \circ }}[\mathrm{T}]=\mathbf{C 1}[\mathrm{N}, \mathrm{T}]$, so we use Theorem 2.1.

${ }^{5}$ The name ' $\mathrm{T}_{\mathrm{q}}$ ' is an abbreviation for 'quasi- $\mathrm{T}$ ', because $(\mathrm{T})$ and $\left(\mathrm{T}_{\mathrm{q}}\right)$ are valid, respectively, in all reflexive and quasi-reflexive Kripke frames. 
Ad 2. By PL and $\mathbf{R}_{\mathbf{P L}}$, the formulas ' $\square \neg r \supset(\square r \supset \square q$ )' and ' $\square q \vee$ $(\square r \supset \diamond r)^{\prime}$ 'belong to $\mathbf{C} 1$. Hence, for any $n \geqslant 0$, also $\left\ulcorner\left(\right.\right.$ alt $\left.\left._{\mathrm{n}}\right) \vee(\mathrm{D}[p / r])\right\urcorner$ and $\left\ulcorner\left(\right.\right.$ Talt $\left.\left._{n}\right) \vee(\mathrm{D}[p / r])\right\urcorner$ belong to $\mathbf{C} 1$. So we use Theorem 2.1.

Finally, note that since $\left(\mathrm{R}^{\diamond}\right)$ belongs to $\mathbf{C} 1$, so we obtain:

Lemma 2.8. For any $\varphi \in{ }^{\mathbf{1}}$ For there is $\varphi^{\mathrm{N}} \in \mathbf{M C N F}$ such that $\ulcorner\varphi \equiv$ $\left.\varphi^{\mathrm{N}}\right\urcorner \in \mathbf{C} 1$ and every conjunct in $\varphi^{\mathrm{N}}$ has one of the following forms:

1. $\left\ulcorner\alpha \vee \bigvee_{i=1}^{k} \square \gamma_{i}\right\urcorner$, where $k \geqslant 0$,

2. $\left\ulcorner\diamond \beta \vee \bigvee_{i=1}^{k} \square \gamma_{i}\right\urcorner$, where $k \geqslant 0$,

3. $\left\ulcorner\alpha \vee \diamond \beta \vee \bigvee_{i=1}^{k} \square \gamma_{i}\right\urcorner$, where $k \geqslant 0$,

4. $\left\ulcorner\bigvee_{i=1}^{k} \square \gamma_{i}\right\urcorner$, where $k>0$.

\subsection{Kripke style semantics for very weak t-normal and t-regular logics}

In $[9,11]$ for very weak t-normal and t-regular modal logics we used simplified Kripke style semantics, i.e., so-called t-normal models and $t$-regular models, respectively. The first ones models we can build on t-normal frames which are pairs of the form $\langle w, A\rangle$, where:

1. $w$ is a «distinguished» world,

2. A is a set of worlds which are alternatives to the world $w$.

For any t-normal frame $\langle w, A\rangle$ we create a t-normal model of the form $\langle w, A, V\rangle$, where:

1. $V$ is a function from For $\times(\{\mathbf{w}\} \cup \mathbf{A})$ to $\{0,1\}$ such that:

(i) for any world $x \in A \cup\{w\}$, the function $V(\cdot, x)$ is a PL-valuation;

(ii) for the world $w$ and any $\varphi \in$ For

$\left(\mathrm{n} V_{w}^{\square}\right) \quad V(\square \varphi, w)=1 \quad$ iff $\quad \forall_{x \in A} V(\varphi, x)=1$.

For any world from $A \backslash\{w\}$ and any $\varphi \in$ For, the formula $\ulcorner\square \varphi\urcorner$ may have an arbitrary value.

Thus, worlds from $A \backslash\{w\}$ are «queer», but the «distinguished» world $w$ is «normal».

A formula $\varphi$ is true in a t-normal model $\langle w, A, V\rangle$ iff $V(\varphi, w)=1$. Next we say that a formula is true in a t-normal frame iff it is true in every t-normal model which is based on this frame. Moreover, for any very weak t-normal logic $\boldsymbol{\Lambda}$ and any class $\mathscr{C}$ of t-normal frames (resp. models) we say that a given formula is valid in $\mathscr{C}$ iff it is true in all members of $\mathscr{C}$. 
We say that a t-normal frame $\langle w, A\rangle$ is self-associate (resp. empty, nonempty) iff $w \in A$ (resp. $A=\varnothing, A \neq \varnothing$ ). For the individual formulas we can assign the respective kinds of frames. We have the following pairs: (Q) to emptiness; (T) to self-associativity; (D) to non-emptiness; (Talt 0 ) to self-associativity or emptiness; $\left(\right.$ al $\left._{\mathrm{n}}\right)$ to $\operatorname{Card} A \leqslant n$; and $\left(\mathrm{Talt}_{\mathrm{n}}\right)$ to the condition that either $w \in A$ or $\operatorname{Card} A \leqslant n$, for any $n>0$.

Let $\mathbf{F}$ (resp. $\mathbf{M}$ ) be the class of all t-normal frames (resp. models). Moreover, let $\mathbf{F}^{\mathbf{s a}}$ (resp. $\mathbf{F}^{\varnothing}, \mathbf{F}^{+}$) be the class of t-normal frames which are self-associate (resp. empty, non-empty). Clearly, $\mathbf{F}^{\text {sa }} \subsetneq \mathbf{F}^{+}, \mathbf{F}^{\varnothing} \cup \mathbf{F}^{+}=\mathbf{F}$, and $\mathbf{F}^{\varnothing} \cap \mathbf{F}^{+}=\varnothing$. Furthermore, for any $n \geqslant 0$, let $\mathbf{F}^{\leqslant n}$ be the class of all t-normal frames in which $\operatorname{Card} A \leqslant n$. Of course, $\mathbf{F}^{\leqslant 0}=\mathbf{F}^{\varnothing}$ and for any $n \geqslant 0$ we have $\mathbf{F}^{\leqslant n} \cap \mathbf{F}^{+}=\mathbf{F}^{\leqslant n} \backslash \mathbf{F}^{\varnothing}$ and $\mathbf{F}^{+} \cap\left(\mathbf{F}^{\mathbf{s a}} \cup \mathbf{F}^{\leqslant n}\right)=\mathbf{F}^{\text {sa }} \cup\left(\mathbf{F}^{\leqslant n} \cap \mathbf{F}^{+}\right)$. Finally, let $\mathbf{M}^{\text {sa }}$ (resp. $\mathbf{M}^{\varnothing}, \mathbf{M}^{+}, \mathbf{M}^{\leqslant n}$ ) be the class of t-normal models which are built on frames from $\mathbf{F}^{\text {sa }}$ (resp. $\mathbf{F}^{\varnothing}, \mathbf{F}^{+}, \mathbf{F}^{\leqslant n}$ ).

In [9] we proved the following determination theorem: ${ }^{6}$

Theorem 2.9. The logics $\mathbf{S 0 . 5 ^ { \circ }}$, S0.5 ${ }^{\circ}[\mathrm{D}]$, S0.5 ${ }^{\circ}\left[\right.$ Talt $\left._{0}\right]$, and $\mathbf{S 0 . 5}$ are determined by the classes $\mathbf{F}, \mathbf{F}^{+}, \mathbf{F}^{\mathbf{s a}} \cup \mathbf{F}^{\varnothing}$, and $\mathbf{F}^{\text {sa }}$, respectively.

By theorems 2.3 and 2.9 we obtain:

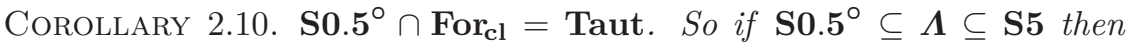
$\Lambda \cap$ For $_{\mathrm{cl}}=$ Taut.

Moreover, by methods used in $[9,11]$, we can get the following generalization of Theorem 2.9:

THEOREM 2.11. Let $\left(\mathrm{X}_{1}\right), \ldots,\left(\mathrm{X}_{\mathrm{k}}\right)$ be any formulas from among the following ones: (T), (D), (Q), (Talt $\left.\mathrm{t}_{0}\right)$, and for any $n>0,\left(\mathrm{alt}_{\mathrm{n}}\right)$ and $\left(\mathrm{Talt}_{\mathrm{n}}\right)$. Then the $t$-normal logic $\mathbf{S 0 . 5 ^ { \circ }}\left[\mathrm{X}_{1} \ldots \mathrm{X}_{\mathrm{k}}\right]$ is determined by the class of all t-normal frames which satisfy all conditions for formulas $\left(\mathrm{X}_{1}\right), \ldots,\left(\mathrm{X}_{\mathrm{k}}\right)$.

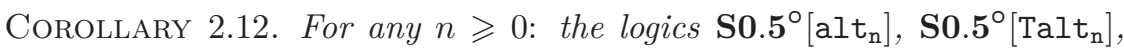
and $\mathbf{S 0 . 5 ^ { \circ }}\left[\mathrm{D}, \mathrm{Talt}_{\mathrm{n}}\right]$ are determined by the classes $\mathbf{F} \leqslant n, \mathbf{F}^{\mathbf{s a}} \cup \mathbf{F} \leqslant n$, and $\mathbf{F}^{\mathrm{sa}} \cup\left(\mathbf{F}^{\leqslant n} \cap \mathbf{F}^{+}\right)$, respectively.

So we also obtain $\mathbf{S 0 . 5}=\mathbf{S 0 . 5}{ }^{\circ}\left[\mathrm{D}, \mathrm{Talt} \mathrm{t}_{0}\right]$, because $\mathbf{F}^{\mathrm{sa}}=\mathbf{F}^{\mathrm{sa}} \cup(\mathbf{F} \leqslant 0 \cap$ $\left.\mathbf{F}^{+}\right)$.

Corollary 2.13. For all $n>0$ and $i \in\{1, \ldots, n\}$ :

$$
\text { S0.5 }^{\circ}\left[\operatorname{Talt}_{\mathrm{n}}\right]=\text { S0.5 }^{\circ}\left[\mathrm{Talt}_{n-i}\right] \cap \mathbf{S 0 . 5}^{\circ}\left[\mathrm{D}, \mathrm{Talt}_{\mathrm{n}}\right] .
$$

\footnotetext{
${ }^{6}$ For the logic S0.5 see also [4, Exercise 11.8].
} 
Proof: For all $n>0$ and $i \in\{1, \ldots, n\}: \varphi \in \mathbf{S 0 . 5}^{\circ}\left[\right.$ Talt $\left._{n-i}\right] \cap \mathbf{S 0 . 5}^{\circ}[\mathrm{D}$, Talt $\left._{\mathrm{n}}\right]$ iff $\varphi$ is true in all t-normal models $\langle w, A, V\rangle$ in which either $w \in A$, or $\operatorname{Card} A \leqslant n-i$, or $0<\operatorname{Card} A \leqslant n$ iff $\varphi$ is true in all t-normal models $\langle w, A, V\rangle$ in which $w \in A$ or $\operatorname{Card} A \leqslant n$ iff $\varphi \in \mathbf{S 0 . 5}^{\circ}\left[\right.$ Talt $\left._{\mathrm{n}}\right]$.

In the case of very weak t-regular logics we broaden the class of tnormal models by the class of queer models of the form $\langle w, V\rangle$ with only one non-normal world $w$ and a valuation $V:$ For $\times\{\mathbf{w}\} \rightarrow\{\mathbf{0}, \mathbf{1}\}$ which satisfies classical conditions for truth-value operators and such that

(ii') for the world $w$ and any $\varphi \in$ For

$$
\left(\mathrm{r} V_{w}^{\square}\right) \quad V(\square \varphi, w)=0 \text {. }
$$

As for t-models, a formula $\varphi$ is true in a queer model $\langle w, V\rangle$ iff $V(\varphi, w)=1$.

Let $\mathbf{q M}$ be the class of all queer models. In [9] we proved the following determination theorem:

Theorem 2.14. The logics $\mathbf{C 1}, \mathbf{D} \mathbf{1}, \mathbf{C 1}\left[\mathrm{Talt}_{0}\right]$, and $\mathbf{E} \mathbf{1}$ are determined by the classes $\mathbf{M} \cup \mathbf{q} \mathbf{M}, \mathbf{M}^{+} \cup \mathbf{q} \mathbf{M}, \mathbf{M}^{\mathbf{s a}} \cup \mathbf{M}^{\varnothing} \cup \mathbf{q} \mathbf{M}$, and $\mathbf{M}^{\mathbf{s a}} \cup \mathbf{q} \mathbf{M}$, respectively.

By theorems 2.3 and 2.14 we obtain:

Corollary 2.15. $\mathbf{C} 1 \cap \mathbf{F o r}_{\mathrm{cl}}=$ Taut, so if $\mathbf{C 1} \subseteq \boldsymbol{\Lambda} \subseteq \mathbf{S 5}$, then $\boldsymbol{\Lambda} \cap$ For $_{\mathrm{cl}}=$ Taut.

By methods used in $[9,11]$, we can get the following generalization of Theorem 2.14:

THEOREM 2.16. Let $\left(\mathrm{X}_{1}\right), \ldots,\left(\mathrm{X}_{\mathrm{k}}\right)$ be any formulas from among the following ones: $(\mathrm{T}),(\mathrm{D}),(\mathrm{Q}),\left(\mathrm{Tal}_{\mathrm{o}}\right)$, and for any $n>0,\left(\mathrm{al} \mathrm{t}_{\mathrm{n}}\right)$ and $\left(\mathrm{Tal}_{\mathrm{n}}\right)$. Then the t-regular logic $\mathbf{C} \mathbf{1}\left[\mathrm{X}_{1} \ldots \mathrm{X}_{\mathrm{k}}\right]$ is determined by the class of models obtained by adding the class $\mathbf{q M}$ to the class of all t-normal models which determined the logic $\mathbf{S 0 . 5 ^ { \circ }}\left[\mathrm{X}_{1} \ldots \mathrm{X}_{\mathrm{k}}\right]$.

Corollary 2.17. For any $n \geqslant 0$ : the logics $\mathbf{C 1}\left[\mathbf{a l t} t_{\mathbf{n}}\right], \mathbf{C 1}\left[\mathrm{Talt}_{\mathrm{n}}\right]$, and $\mathbf{C 1}\left[\mathrm{D}, \mathrm{Talt}_{\mathrm{n}}\right]$ are determined by the classes $\mathbf{M} \leqslant n \cup \mathbf{q M}, \mathbf{M}^{\text {sa }} \cup \mathbf{M} \leqslant n \cup \mathbf{q M}$, and $\mathbf{M}^{\mathbf{s a}} \cup\left(\mathbf{M} \leqslant n \cap \mathbf{M}^{+}\right) \cup \mathbf{q} \mathbf{M}$, respectively.

So we also obtain $\mathbf{E} \mathbf{1}=\mathbf{C} \mathbf{1}\left[\mathrm{D}\right.$, Talt $\left.t_{0}\right]$, because $\mathbf{M}^{\mathbf{s a}}=\mathbf{M}^{\text {sa }} \cup\left(\mathbf{M} \leqslant 0 \cap \mathbf{M}^{+}\right)$.

Similarly as Corollary 2.13 we obtain:

Corollary 2.18. For all $n>0$ and $i \in\{1, \ldots, n-1\}$ :

$$
\mathbf{C 1}\left[\text { Talt }_{\mathbf{n}}\right]=\mathbf{C 1}\left[\text { Talt }_{\mathbf{n}-\mathbf{i}}\right] \cap \mathbf{C 1}\left[\mathrm{D}, \text { Talt }_{\mathbf{n}}\right] .
$$

Regarding t-regular logics, the following facts are interesting (see also Fact 2.5): 
FACT 2.19. For any t-regular logic $\boldsymbol{\Lambda}$ :

1. (N) $\in \Lambda$ iff $\mathbf{S 0 . 5}^{\circ} \subseteq \Lambda$ iff ${ }^{1} \mathrm{SO}^{\circ} \subseteq{ }^{\mathbf{1}} \boldsymbol{\Lambda}$.

2. If $(\mathrm{N}) \in \boldsymbol{\Lambda}$ then ${ }^{\mathbf{1}} \boldsymbol{\Lambda} \nsubseteq \mathbf{E} \mathbf{1}$.

Moreover, if $\boldsymbol{\Lambda}$ is included in $\mathbf{S 5}$, then:

3. If ${ }^{\mathbf{1}} \boldsymbol{\Lambda} \nsubseteq \mathbf{E} \mathbf{1}$ then $(\mathrm{N}) \in \boldsymbol{\Lambda}$.

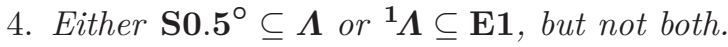

Proof: Ad 1. First, we have: $(\mathrm{N}) \in \boldsymbol{\Lambda}$ iff $\mathbf{S 0 . 5 ^ { \circ }} \subseteq \boldsymbol{\Lambda}$. Second, we use Fact 2.5.

Ad 2. Because $(\mathrm{N}) \notin \mathbf{E} 1$.

Ad 3. Suppose that ${ }^{\mathbf{1}} \boldsymbol{\Lambda} \nsubseteq \mathbf{E} \mathbf{1}$ and $\boldsymbol{\Lambda} \subseteq \mathbf{S 5}$. Then there is $\varphi \in{ }^{\mathbf{1}} \boldsymbol{\Lambda}$ such that $\varphi \notin \mathbf{E} 1$. Hence, by Theorem 2.14, $\varphi$ is false in some model from $\mathbf{M}^{\text {sa }} \cup \mathbf{q M}$. But, by Theorem $2.9, \varphi$ is true in all models from $\mathbf{M}^{\text {sa }}$, since $\varphi \in{ }^{1} \boldsymbol{\Lambda},{ }^{1} \boldsymbol{\Lambda} \subseteq{ }^{1} \mathbf{S} 5={ }^{1} \mathbf{S} \mathbf{0 . 5}$. Therefore $\varphi$ is false in some queer model from qM of the form $\mathfrak{M}^{\varphi}=\left\langle w^{\varphi}, V^{\varphi}\right\rangle$ with only one (non-normal) world $w^{\varphi}$ and a valuation $V^{\varphi}$ : For $\times\left\{\mathbf{w}^{\varphi}\right\} \rightarrow\{\mathbf{0}, \mathbf{1}\}$ satisfying conditions for truth-value operators and such that $V^{\varphi}\left(\diamond \varphi, w^{\varphi}\right)=1$, for any $\varphi \in$ For. In MCNF (see p. 115) there is a formula $\varphi^{\mathrm{N}}:=\left\ulcorner\bigwedge_{i=1}^{c} \kappa_{i}^{\varphi}\right\urcorner$ such that $\left\ulcorner\varphi^{\mathrm{N}} \equiv \varphi\right\urcorner \in \mathbf{C} \mathbf{1}$ and every conjunct of $\varphi^{\mathrm{N}}$ belongs to ${ }^{1} \boldsymbol{\Lambda}$ and has one of the forms (1)-(4) given in Lemma 2.8. Since $\varphi^{N} \in{ }^{1} \Lambda$ and $\mathfrak{M}^{\varphi} \not \models \varphi^{N}$, so there is $\kappa_{*} \in\left\{\kappa_{1}^{\varphi}, \ldots, \kappa_{c}^{\varphi}\right\}$ such that $\kappa_{*} \in{ }^{\mathbf{1}} \boldsymbol{\Lambda}$ and $\mathfrak{M}^{\varphi} \not \models \kappa_{*}$. So we obtain that $\kappa_{*}$ has the form $\left\ulcorner\alpha \vee \bigvee_{i=1}^{k} \square \gamma_{i}\right\urcorner$ with $k \geqslant 0$, or $\left\ulcorner\bigvee_{i=1}^{k} \square \gamma_{i}\right\urcorner$ with $k>0$. So, by Lemma 2.2(1,4), either $\alpha \in$ Taut or there is an $i \in\{1, \ldots, k\}$ such that $\gamma_{i} \in$ Taut. But $\alpha \notin$ Taut, since $\mathfrak{M}^{\varphi} \not \models \kappa_{*}$. Therefore for some $j \in\{1, \ldots, k\}$ we have $\gamma_{j} \in$ Taut. Moreover, $\alpha \notin \boldsymbol{\Lambda}$. So $\kappa_{*}$ has the form $\left\ulcorner\alpha \vee \bigvee_{i=1}^{k} \square \gamma_{i}\right\urcorner$ or $\left\ulcorner\bigvee_{i=1}^{k} \square \gamma_{i}\right\urcorner$ with $k>0$ in both cases. In the first case there is a uniform substitution $s$ such that $\ulcorner s(\alpha) \equiv$ $\neg \alpha\urcorner \in$ Taut. Since $\left\ulcorner\neg \alpha \vee \bigvee_{i=1}^{k} \square s\left(\gamma_{i}\right)\right\urcorner \in \boldsymbol{\Lambda}$, so in the first case also $\kappa_{*}^{\prime}:=\left\ulcorner\bigvee_{i=1}^{k} \square \gamma_{i} \vee \bigvee_{i=1}^{k} \square s\left(\gamma_{i}\right)\right\urcorner \in \boldsymbol{\Lambda}$. Note that $\ulcorner\square \chi \supset \mathrm{N}\urcorner \in \boldsymbol{\Lambda}$, for any $\chi \in$ For. So in the first (resp. second) case we have $\left\ulcorner\kappa_{*}^{\prime} \supset \mathrm{N}\right\urcorner \in \boldsymbol{\Lambda}$ (resp. $\left.\left\ulcorner\kappa_{*} \supset \mathrm{N}\right\urcorner \in \boldsymbol{\Lambda}\right)$. Hence in the both cases, $(\mathrm{N}) \in \boldsymbol{\Lambda}$.

Ad 4. First, by items 1 and 3 . Second, since ${ }^{1}$ S0.5 ${ }^{\circ} \nsubseteq \mathbf{E} 1$.

By definitions and the above facts we obtain the following corollaries:

Corollary 2.20. 1. (T) $\notin \mathbf{C 1}\left[\mathrm{D}, \mathrm{Talt}_{1}\right] \subsetneq \mathrm{S} .5^{\circ}\left[\mathrm{D}, \mathrm{Talt}_{1}\right]$.

2. (D), (T) $\notin \mathbf{C 1}\left[\mathrm{Talt}_{0}\right] \subsetneq \mathbf{S} 0.5^{\circ}\left[\mathrm{Talt}_{0}\right]$.

3. For any $n \geqslant 0$ : $\left(\right.$ Talt $\left._{\mathrm{n}}\right) \notin \mathbf{C 1}\left[\mathrm{D}, \mathrm{Talt}_{\mathrm{n}+1}\right] \subsetneq \mathbf{S 0 . 5 ^ { \circ }}\left[\mathrm{D}, \mathrm{Talt}_{\mathrm{n}+1}\right]$.

4. For any $n \geqslant 0$ : $\left(\right.$ Talt $\left._{\mathrm{n}}\right) \notin \mathbf{C 1} \subsetneq \mathbf{S 0 . 5 ^ { \circ }}$. 
Corollary 2.21. For any $n \geqslant 0$ :

1. $\mathbf{C} 1 \subsetneq \mathbf{C 1}\left[\mathrm{Talt}_{\mathrm{n}+1}\right] \subsetneq \mathbf{C 1}\left[\mathrm{Talt}_{\mathrm{n}}\right] \subsetneq \mathbf{E} \mathbf{1}$.

2. S0.5 ${ }^{\circ} \subsetneq \mathbf{S} 0.5^{\circ}\left[\mathrm{Talt}_{\mathrm{n}+1}\right] \subsetneq \mathbf{S} 0.5^{\circ}\left[\mathrm{Talt}_{\mathrm{n}}\right] \subsetneq \mathbf{S 0 . 5}$.

Moreover, by the above corollaries and Fact 2.7, we have (see Figure 1): Corollary 2.22. For any $n \geqslant 0$ :

1. C1 $\left[\mathrm{Talt}_{\mathrm{n}}\right] \subsetneq \mathbf{C 1}\left[\mathrm{D}, \mathrm{Talt}_{\mathrm{n}}\right]$ and ${ }^{\mathbf{1}} \mathrm{C} 1\left[\mathrm{Talt}_{\mathrm{n}}\right] \subsetneq{ }^{\mathbf{1}} \mathrm{C} 1\left[\mathrm{D}, \mathrm{Talt}_{\mathrm{n}}\right]$.

2. S0.5 ${ }^{\circ}\left[\mathrm{Talt}_{\mathrm{n}}\right] \subsetneq \mathrm{SO}^{\circ} \mathbf{5}^{\circ}\left[\mathrm{D}, \mathrm{Talt}_{\mathrm{n}}\right]$ and ${ }^{1} \mathrm{SO.5}^{\circ}\left[\mathrm{Talt}_{\mathrm{n}}\right] \subsetneq{ }^{1} \mathrm{SO} .5^{\circ}\left[\mathrm{D}, \mathrm{Talt}_{\mathrm{n}}\right]$.

3. ${ }^{1} \mathrm{C} 1 \subsetneq{ }^{1} \mathbf{C} 1\left[\mathrm{Talt}_{\mathrm{n}+1}\right] \subsetneq{ }^{1} \mathrm{C} 1\left[\mathrm{Talt}_{\mathrm{n}}\right] \subsetneq{ }^{1} \mathbf{E} 1$.

4. ${ }^{1} \mathrm{~S} 0.5^{\circ} \subsetneq{ }^{1} \mathrm{~S} 0.5^{\circ}\left[\mathrm{Talt}_{\mathrm{n}+1}\right] \subsetneq{ }^{1} \mathrm{SO}^{\circ}$ ( $\left.^{\circ} \mathrm{Talt}_{\mathrm{n}}\right] \subsetneq{ }^{1} \mathrm{~S} 0.5$.

5. $\mathbf{C} 1 \subsetneq \mathbf{D} 1 \subsetneq \mathbf{C 1}\left[\mathrm{D}, \mathrm{Talt}_{\mathrm{n}+1}\right] \subsetneq \mathbf{C} 1\left[\mathrm{D}, \mathrm{Talt}_{\mathrm{n}}\right]$ and ${ }^{1} \mathrm{C} 1 \subsetneq{ }^{1} \mathrm{~S} 0.5^{\circ}[\mathrm{D}] \subsetneq{ }^{1} \mathrm{C} 1\left[\mathrm{D}, \mathrm{Talt}_{\mathrm{n}+1}\right] \subsetneq{ }^{1} \mathrm{C} 1[\mathrm{D}, \mathrm{Talt} \mathrm{n}]$.

6. S0.5 ${ }^{\circ} \subsetneq \mathbf{S 0 . 5 ^ { \circ }}[\mathrm{D}] \subsetneq \mathbf{S 0 . 5 ^ { \circ }}\left[\mathrm{D}, \mathrm{Talt}_{\mathrm{n}+1}\right] \subsetneq \mathbf{S 0 . 5 ^ { \circ }}\left[\mathrm{D}, \mathrm{Talt}_{\mathrm{n}}\right]$ and

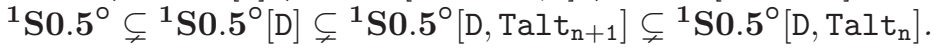

7. ${ }^{1} \mathbf{D} 1 \nsubseteq \mathbf{C} 1\left[\mathrm{Talt}_{0}\right]$ and ${ }^{1} \mathbf{C} 1\left[\mathrm{Talt}_{0}\right] \not \mathbf{C} 1\left[\mathrm{D}, \mathrm{Talt}_{1}\right]$.

8. ${ }^{1} \mathbf{S 0 . 5}{ }^{\circ}[\mathrm{D}] \nsubseteq \mathbf{S 0 . 5}^{\circ}\left[\mathrm{Talt}_{0}\right]$ and ${ }^{1} \mathrm{S0.5}^{\circ}\left[\mathrm{Talt}_{0}\right] \nsubseteq \mathbf{S 0 . 5}^{\circ}\left[\mathrm{D}, \mathrm{Talt}_{1}\right]$.

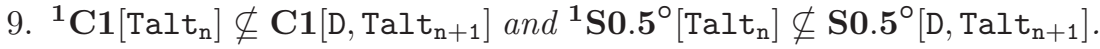

10. ${ }^{1} \mathrm{C} 1={ }^{1} \mathrm{D} 1 \cap{ }^{1} \mathrm{C} 1\left[\mathrm{Talt} \mathrm{t}_{\mathrm{n}}\right]$ and ${ }^{1} \mathrm{~S} 0.5^{\circ}={ }^{1} \mathrm{~S} 0.5^{\circ}[\mathrm{D}] \cap{ }^{1} \mathrm{~S} 0.5^{\circ}\left[\mathrm{Talt}_{\mathrm{n}}\right]$.

\section{Pollack's results and their extension}

In [12] Pollack analyzed a certain set B which he called the theory of "Basic Modal Logic". Pollack defined B as the set of all formulas from ${ }^{\mathbf{1}}$ For which are $\mathbf{S 5}$-valid, i.e., $\mathrm{B}:={ }^{\mathbf{1}} \mathbf{S} 5$. Pollack proved:

TheOrem 3.1 ([12, Th. 3]). B is the smallest subset of ${ }^{\mathbf{1}}$ For such that:

1. it includes the set ${ }^{\mathbf{1}} \mathbf{P L}$,

2. it includes the set $\square$ Taut,

3. it is closed under the rule of detachment for material implication,

4. it contains all formulas from ${ }^{\mathbf{1}}$ For which are instances of the formulas $(\mathrm{K})$ and $(\mathrm{T})$.

Theorem 3.2 ([12, Th. 4]). B $={ }^{\mathbf{1}} \mathbf{S} \mathbf{1}={ }^{\mathbf{1}} \mathbf{S 2}={ }^{\mathbf{1}} \mathbf{S} 3={ }^{\mathbf{1}} \mathbf{S} 4={ }^{\mathbf{1}} \mathbf{T}$.

Theorem 3.3 ([12, Th. 6]). For any logic $\boldsymbol{\Lambda}$ : if $\mathbf{S 5} \subsetneq \boldsymbol{\Lambda}$, then $\mathrm{B} \subsetneq{ }^{\mathbf{1}} \boldsymbol{\Lambda}$.

But Pollack did not notice that also:

Theorem 3.4. 1. $\mathrm{B}={ }^{\mathbf{1}} \mathbf{S} \mathbf{0 . 5}$.

2. For any modal logic $\boldsymbol{\Lambda}$ included in $\mathbf{S 5 :} \mathrm{B}={ }^{\mathbf{1}} \boldsymbol{\Lambda}$ iff $\mathrm{B} \subseteq{ }^{\mathbf{1}} \boldsymbol{\Lambda}$ iff $\mathbf{S 0 . 5} \subseteq \boldsymbol{\Lambda}$. Thus, the theory $\mathrm{B}$ is assigned to all modal logics between $\mathbf{S 0 . 5}$ and $\mathbf{S 5}$. 


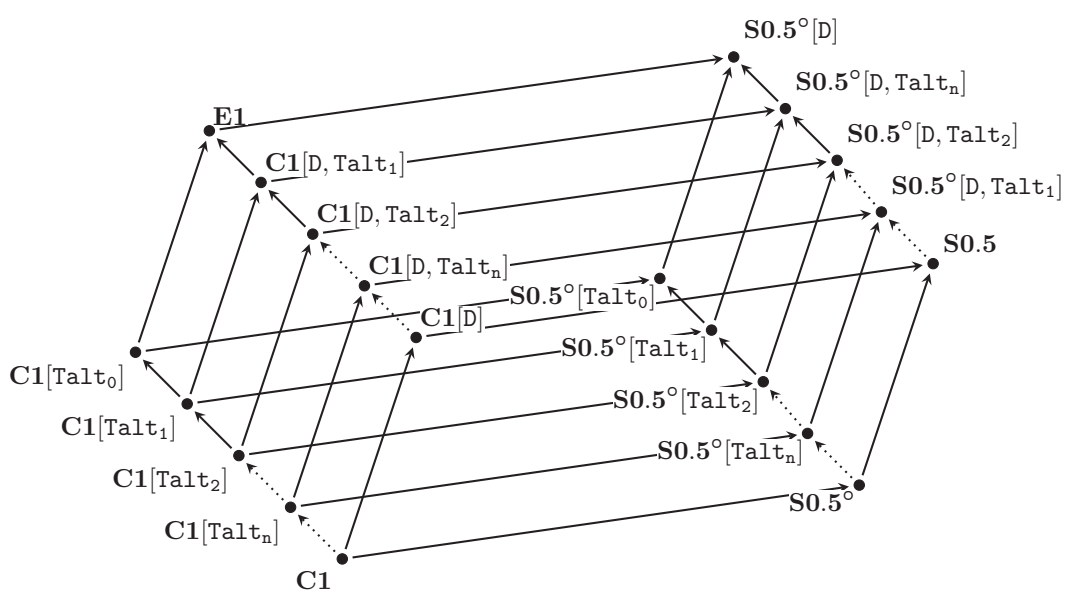

Fig. 1. The lattice of selected very weak t-regular and very weak t-normal $\operatorname{logics}$

Proof: Ad 1. By definition of S0.5 and Theorem 3.1, we have $\mathrm{B} \subseteq \mathbf{S 0 . 5} \subseteq$ S5. So $\mathrm{B} \subseteq{ }^{1} \mathrm{~S} 0.5 \subseteq{ }^{1} \mathrm{~S} 5=\mathrm{B}$.

Ad 2. Suppose that $\boldsymbol{\Lambda} \subseteq \mathbf{S 5}$. Then ${ }^{\mathbf{1}} \boldsymbol{\Lambda} \subseteq{ }^{\mathbf{1}} \mathbf{S} 5=\mathrm{B}$. So: $\mathrm{B} \subseteq{ }^{\mathbf{1}} \boldsymbol{\Lambda}$ iff ${ }^{1} \boldsymbol{\Lambda}=\mathrm{B}$. Moreover, if $\mathrm{B} \subseteq{ }^{\mathbf{1}} \boldsymbol{\Lambda}$, then $\square$ Taut $\cup\{\mathrm{K}, \mathrm{T}\} \subseteq \boldsymbol{\Lambda}$, by Theorem 3.1. So S0.5 $\subseteq \Lambda$. Finally, if S0.5 $\subseteq \Lambda$, then $\mathrm{B}={ }^{1}$ S0.5 $\subseteq{ }^{1} \Lambda \subseteq{ }^{1} \mathrm{~S} 5=\mathrm{B}$.

Note that, by Theorem 3.4, we can apply Lemma 2.2 also for the logic S0.5.

In the present paper we generalize Pollack's result in a few ways. First, we show that there are countably many basic theories («Pollack style theories of basic modal logics») which we can assign to modal logics between C1 and S5. Moreover, we show that these theories are linked with certain t-regular and t-normal modal logics (see Theorem 4.1 and figures 1 and 2). Second, we will describe relations that hold between basic theories (see Theorem 4.2). Third, for every logic between C1 and S5 we assign a basic theory which corresponds to it (see theorems 6.1 and 6.2 in Part 2). Fourth, we will indicate a relationship between some normal logics and basic theories (see Figure 3 in Part 2). 


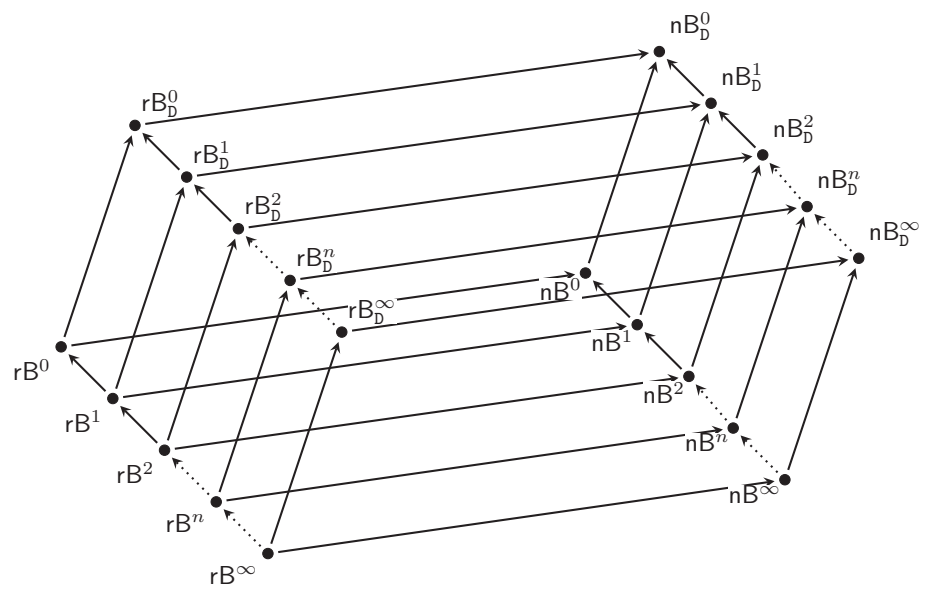

Fig. 2. The lattice of all "basic theories" (corresponding to logics from Figure 1)

\section{4. "Basic theories" for modal logics between C1 and S5}

For all modal logics between $\mathbf{C} 1$ and $\mathbf{S 5}$ we will describe certain basic theories. Each of them will be the smallest subset of the set ${ }^{\mathbf{1}}$ For that fulfil some specific conditions. Thus, we will build two groups of «basic theories». The first one will be connected with t-normal logics and normal logics. The second one will be connected with properly t-regular logics and properly regular logics.

The first group consists of the following "normal basic theories" satisfying conditions (1)-(3), and some modification of condition (4) from Theorem 3.1. First, for any $n \geqslant 0$ we can build the following two kinds of such theories:

1. for $n B_{D}^{n}$ : in (4) instead of (T) we will use both (D) and (Talt $\left.t_{n}\right)$

2. for $n B^{n}$ : in (4) instead of $(T)$ we will use $\left(\right.$ Talt $\left._{n}\right)$.

Note that we have $n B_{D}^{1}=B$, since $\mathbf{S 0 . 5}=\mathbf{S 0 . 5 ^ { \circ }}\left[D\right.$, Talt $\left._{0}\right]$. Second we build the following two "normal basic theories":

1. for $n B_{D}^{\infty}$ : in (4) instead of (T) we will use (D);

2. for $n B^{\infty}$ : in (4) we reject the formula $(T)$. 
Remark 4.1. In [12] Pollack used the identity 'B $:={ }^{\mathbf{1}} \mathbf{S} 5$ ' as the definition of the set B and received Theorem 3.1. In the present paper, we use appropriate versions of the formulation given in Theorem 3.1 as definitions of theories $\mathrm{nB}^{\infty}, \mathrm{nB}_{\mathrm{D}}^{\infty}, \mathrm{nB}^{n}$, and $\mathrm{nB}_{\mathrm{D}}^{n}$ (for $n \geqslant 0$ ). However, we will show that in essence there is no difference between both ways of defining of these sets, because the following equalities hold: $\mathrm{nB}^{\infty}={ }^{1} \mathrm{SO}^{\circ} \mathbf{5}^{\circ}={ }^{1} \mathrm{~K} 4 \mathbf{5}=$ ${ }^{1} \mathrm{~KB}, \mathrm{nB}_{\mathrm{D}}^{\infty}={ }^{1} \mathrm{S0.5}{ }^{\circ}[\mathrm{D}]={ }^{1} \mathrm{KD} 45={ }^{1} \mathrm{KDB}$, and for any $n \geqslant 0$ both $\mathrm{nB}^{n}={ }^{1} \mathrm{SO.5}^{\circ}\left[\right.$ Talt $\left._{\mathrm{n}}\right]={ }^{1} \mathrm{~K} 45 \oplus\left(\right.$ Talt $\left._{\mathrm{n}}\right)$ and $\mathrm{nB}_{\mathrm{D}}^{n}={ }^{1} \mathrm{SO}^{\circ}{ }^{\circ}\left[\mathrm{D}\right.$, Talt $\left._{\mathrm{n}}\right]=$ ${ }^{1} \mathrm{KD} 45 \oplus\left(\mathrm{Talt}_{\mathrm{n}}\right)$ (see Theorem 4.1). Moreover, $\mathrm{nB}_{\mathrm{D}}^{1}={ }^{1} \mathrm{~S} 0.5^{\circ}\left[\mathrm{D}, \mathrm{Talt}_{0}\right]=$ ${ }^{1} \mathrm{~S} 0.5={ }^{1} \mathrm{KD} 45 \oplus\left(\mathrm{Talt}_{0}\right)={ }^{1} \mathrm{~S} 5=$ : B.

The second group consists of the following "regular basic theories" satisfying conditions (1) and (3), and some modification of the conditions (2) and (4). Generally, in (2) instead of the set $\square$ Taut we use the set $\mathbf{M}_{\text {Taut }}$. Moreover, first, for any $n \geqslant 0$, we can build the following two kinds of such theories:

1. for $r B_{D}^{n}$ : in (4) instead of (T) we will use both (D) and $\left(\right.$ Tal $\left._{n}\right)$;

2. for $r B^{n}$ : in (4) instead of $(\mathrm{T})$ we will use $\left(\mathrm{Talt}_{\mathrm{n}}\right)$.

Second we build the following two "regular basic theories":

1. for $r B_{D}^{\infty}$ : in (4) instead of ( $T$ ) we will use (D);

2. for $r B^{\infty}$ : in (4) we reject the formula $(T)$.

In the light of the definitions of "basic theories", the definitions of appropriate logics, and Theorem 3.4 we obtain:

TheOREM 4.1. For any $n \geqslant 0$ :

1. $\mathrm{nB}^{n}={ }^{1} \mathrm{SO.5}^{\circ}\left[\mathrm{Talt}_{\mathrm{n}}\right]$ and $\mathrm{nB}_{\mathrm{D}}^{n}={ }^{1} \mathrm{~S} 0.5^{\circ}\left[\mathrm{D}, \mathrm{Talt}_{\mathrm{n}}\right]$. So $\mathrm{nB}_{\mathrm{D}}^{1}=\mathrm{B}=$ ${ }^{1} \mathrm{~S} 0.5$.

2. $\mathrm{rB}^{n}={ }^{1} \mathrm{C} 1\left[\mathrm{Talt}_{\mathrm{n}}\right]$ and $\mathrm{rB}_{\mathrm{D}}^{n}={ }^{1} \mathrm{C} 1\left[\mathrm{D}, \mathrm{Talt}_{\mathrm{n}}\right]$.

3. $\mathrm{nB}^{\infty}={ }^{1} \mathrm{~S} .5^{\circ}$ and $\mathrm{nB}_{\mathrm{D}}^{\infty}={ }^{1} \mathrm{~S} 0.5^{\circ}[\mathrm{D}]$.

4. $\mathrm{rB}^{\infty}={ }^{1} \mathrm{C} 1$ and $r \mathrm{~B}_{\mathrm{D}}^{\infty}={ }^{1} \mathrm{C} 1[\mathrm{D}]={ }^{\mathbf{1}} \mathrm{D} 1$.

In the light of the above theorem and Corollary 2.22 we obtain the following theorem, which expresses dependencies between basic theories (see figures 1 and 2).

TheOREM 4.2. For both cases $x=\mathrm{n}$ and $x=\mathrm{r}$ for any $n \geqslant 0$ we have:

1. $x \mathrm{~B}^{\infty} \subsetneq x \mathrm{~B}_{\mathrm{D}}^{\infty}$ and $x \mathrm{~B}^{n} \subsetneq x \mathrm{~B}_{\mathrm{D}}^{n}$.

2. $x \mathrm{~B}_{\mathrm{D}}^{\infty} \subsetneq x \mathrm{~B}_{\mathrm{D}}^{n+1} \subsetneq x \mathrm{~B}_{\mathrm{D}}^{n} \subseteq \mathrm{B}$. 
3. $x \mathrm{~B}^{\infty} \subsetneq x \mathrm{~B}^{n+1} \subsetneq x \mathrm{~B}^{n} \subsetneq \mathrm{B}$.

4. $x \mathrm{~B}_{\mathrm{D}}^{\infty} \nsubseteq x \mathrm{~B}^{0}$ and $x \mathrm{~B}^{n} \nsubseteq x \mathrm{~B}_{\mathrm{D}}^{n+1}$.

5. $x \mathrm{~B}^{\infty}=x \mathrm{~B}_{\mathrm{D}}^{\infty} \cap x \mathrm{~B}^{n}$.

6. For any $k \in\{0, \ldots, n\}: \mathrm{B}^{n}=x \mathrm{~B}_{\mathrm{D}}^{n} \cap x \mathrm{~B}^{n-k}$.

Moreover, $\mathrm{rB}^{\infty} \subsetneq \mathrm{nB}^{\infty}, \mathrm{rB}_{\mathrm{D}}^{\infty} \subsetneq \mathrm{nB}_{\mathrm{D}}^{\infty}, \mathrm{rB}^{n} \subsetneq \mathrm{nB}^{n}$, and $\mathrm{rB}_{\mathrm{D}}^{n} \subsetneq \mathrm{nB}_{\mathrm{D}}^{n}$.

Acknowledgements. The author is grateful to prof. Marek Nasieniewski for his valuable suggestions and corrections and to dr Matthew Carmody for linguistic advice and proofreading. This work was supported by funds of the National Science Centre, Poland (no. 2016/23/B/HS1/00344).

\section{References}

[1] B. F. Chellas, Modal Logic. An Introduction, Cambridge University Press: Cambridge, 1980.

[2] B. F. Chellas and K. Segerberg, Modal logics in the vicinty of S1, Notre Dame Journal of Formal Logic 37 (1) (1996), pp. 1-24.

[3] R. A. Girle, $S 1 \neq S 0.9$, Notre Dame Journal of Formal Logic 16 (1975), pp. 339-344.

[4] G. E. Hughes and M. J. Cresswell, A New Introduction to Modal Logic, Routledge: London and New York, 1996.

[5] S. A. Kripke, Semantical analisis of modal logic. II: Non-normal modal propositional calculi, [in:] J. W. Addison, L. Henkin, and A. Tarski (eds.), The Theory of Models. Proc. of the 1963 International Symposium at Berkeley, pp. 206-220, North Holland: Amsterdem, 1965.

[6] E. J. Lemmon, New fundations for Lewis modal systems, The Journal of Symbolic Logic 22 (2) (1957), pp. 176-186. DOI: 10.2307/2964179

[7] E. J. Lemmon, Algebraic semantics for modal logics I, The Journal of Symbolic Logic 31 (1) (1966), pp. 46-65. DOI: 10.2307/2270619

[8] E. J. Lemmon, in collaboration with D. Scott, „Lemmon Notes”: An Introduction to Modal Logic, edited by K. Segerberg, no. 11 in the American Philosophical Quarterly Monograph Series, Basil Blackwell: Oxford, 1977.

[9] A. Pietruszczak, Simplified Kripke style semantics for some very weak modal logics, Logic and Logical Philosophy 18 (3-4) (2010), pp. 271-296. DOI: 10.12775/LLP.2009.013 
[10] A. Pietruszczak, Semantical investigations on some weak modal logics. Part I, Bulletin of the Section of Logic 41 (1/2) (2012), pp. 33-50.

[11] A. Pietruszczak, Semantical investigations on some weak modal logics. Part II, Bulletin of the Section of Logic 41 (3/4) (2012), pp. 109-130.

[12] J. L. Pollack, Basic Modal Logic, The Journal of Symbolic Logic 32 (3) (1967), pp. 355-365. DOI: $10.2307 / 2270778$

[13] G. Priest, An Introduction to Non-Classical Logic, 2th edition, Cambridge University Press, 2008. DOI: 10.1017/CBO9780511801174

[14] R. Routley, Decision procedure and semantics for C1, E1 and S0.5\%, Logique et Analyse 44 (1968), pp. 468-471.

[15] K. Segerberg, An Essay in Classical Modal Logic, vol. I and vol. II, Uppsala, 1971.

[16] G. Takeuti, Proof Theory, North-Holland: Amsterdam, 1975.

[17] M. Zakharyaschev, F. Wolter, and A. Chagrov, Advanced modal logic, in D. M. Gabbay and F. Guenthner (eds.), Handbook of Philosophical Logic, 2nd Edition, Volume 3, pp. 83-266. Kluwer Academic Publishers, 2001. DOI: $10.1007 / 978-94-017-0454-0$

Department of Logic

Nicolaus Copernicus University in Torun

ul. Moniuszki 16, 87-100 Toruń, Poland

e-mail: Andrzej.Pietruszczak@umk.pl 